\title{
A Computationally Efficient Approach to Microwave Circuit Modeling of Complex High Temperature Superconductor Circuits
}

\author{
Jeffrey M. Pond \\ Naval Research Laboratory, Washington, DC 20375-5000
}

\begin{abstract}
A computationally efficient approach to modeling complex high temperature superconductor (HTS) microwave circuits is described which combines two-dimensional electromagnetic solutions of microwave transmission lines, incorporating a phenomenological description of HTS behavior, with conventional commercially available microwave-circuit computer-aided-design software. Internal inductance effects are known to have a substantial impact on the design and implementation of narrowband HTS circuits. Phase velocities and characteristic impedances can be well modeled using a phenomenological description of HTS superconductivity along with a generalized approximate boundary condition and a two dimensional electromagnetic solution to the waveguiding structure. Resultant parameterized design equations can then be incorporated into standard commercially available microwave-circuit computer-aided-design software. The test case examined in detail was the superconducting channelized receiver delivered by the Naval Research Laboratory to Phase II of the High Temperature Superconducting Space Experiment. Excellent agreement was obtained between measurements made on the demultiplexer and this HTS microwave circuit modeling approach.
\end{abstract}

\section{INTRODUCTION}

Accurate and efficient modeling of superconducting microwave circuits is needed in order to fully exploit the advantages of high-temperature superconductors (HTS) for microwave applications. One of the principle microwave application areas that should benefit from HTS technology is that of very narrow bandwidth $(<1 \%)$ filters and multiplexers. Although strongly dependent on a number of factors, narrowband thin-film-based distributed filters fabricated using HTS as opposed to normal metals can have insertion losses of approximately $1 \mathrm{~dB}$ instead of more than $10 \mathrm{~dB}$ when the bandwidth is small [1]. In order to fabricate such a filter and have it perform according to design, it is necessary to be able to design the resonant elements and their interactions to very tight tolerances. The technique described provides a convenient means of achieving the necessary design accuracy.

Microwave computer-aided design (CAD) of narrowband circuits is not an easy task for several reasons. Microwave CAD can be subdivided into two major categories: circuitmodel based, and electromagnetic-field-simulation based. The first is capable of dealing with large structures and complicated topologies, but the accuracy is limited by the accuracy of the circuit element models. For circuit-based sim-

Manuscript received August 27, 1996.

This work was supported, in part, by the Office of Naval Research. ulators, many of the difficulties are related to the inability to account for the effects that the superconductor has on the circuit element response. This is most often observed as a temperature-dependent shift in the phase length of a circuit element due to the internal energy of the superconductor. Although generally small compared to the external energy stored, the effect is sufficient to change the resonant frequency by as much as several percent. As for electromagnetic field solvers, practical computational considerations limit their use to relatively simple geometries, especially in the case of full three dimensional simulators which also must model the fields internal to the superconducting films.

Recently, there has been considerable attention in the literature to addressing the issue of modeling plana superconducting microwave circuits. Several studies have followed the approach of numerically solving for the fields both internal as well as external to the superconductor and have had good success in modeling the response of superconducting transmission lines [2]-[5]. Another approach is to consider those cases where the superconductor can be accounted for by invoking a boundary condition which accounts for the internal properties of the superconductor and its thickness without explicitly solving for the internal fields [6]-[8]. This approach was first applied to the spectral domain approach in the case of very thin superconducting films where the approximate boundary condition is well defined [6]. Subsequently, a correction to the approximate boundary condition which allows the spectral domaln approach to be applied in the case of arbitrarily thick films has been employed [8]. Since it is only necessary to solve for the external fields, the computational requirements are greatly reduced.

In the next section an approach is presented which stresses computational efficiency by employing electromagnetic modeling only when needed and only to provide parameterized corrections to standard circuit model CAD. This approach is then applied successfully to model the measured response of the HTS demultiplexer portion of the channelized receiver built by the Naval Research Laboratory for the High Temperature Superconductivity Space Experiment (HTSSE).

\section{MULTIPLEXER AND MEASURED RESULTS}

The experimental results for the HTS channelized receiver have been discussed in detail [9]. The receiver consists of a four channel demultiplexer based on a modified logarithmic periodic design operating at $\sim 9.5 \mathrm{GHz}$ with each passbanc $\sim 100 \mathrm{MHz}$ in width. The circuit was implemented on a 254 $\mu \mathrm{m}$-thick $\mathrm{MgO}$ substrate onto which $\mathrm{YBa}_{2} \mathrm{Cu}_{3} \mathrm{O}_{7-\delta}$ wa: deposited on both sides via pulsed laser deposition. Each o the four channelized signals was then downconverted usin: commercially available microwave monolithic integrater 
circuit mixers. Two external local oscillator (LO) signals were supplied which where selected to provide frequencies to fall between non-adjacent, symmetrically located channels. This results in two upper-sideband and two lower-sideband intermediate frequency (IF) responses. The LO signals were delivered to the downconverters via two in-phase power splitters which were implemented in conventional microstrip. Each of the four intermediate frequencies were then detected with diode detectors. In order to measure the response at microwave frequencies, prior to incorporating the mixers, the outputs of all four multiplexer channels were routed backwards through the local oscillator power splitters which act as combiners. Thus it was possible to characterize the HTS multiplexer response, albeit embedded in the combiner response, at microwave frequencies with a network analyzer. The measured microwave responses of the four channel demultiplexer cascaded through the two power combiner channels are shown in Fig. 1 at temperatures around the $77 \mathrm{~K}$ operating temperature of the HTSSE experiment. Approximately $3 \mathrm{~dB}$ of the insertion loss is directly attributable to the inherent design of the power splitters.

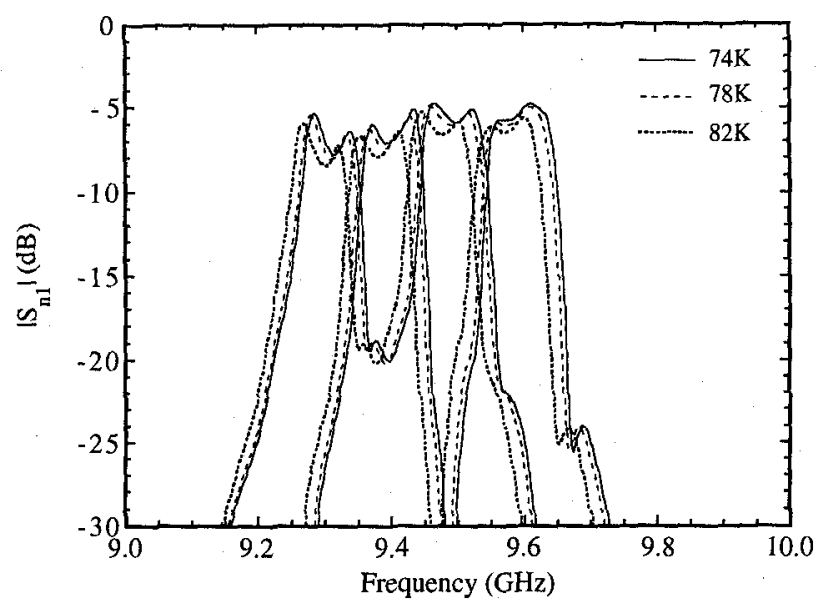

Fig. 1. Measured insertion loss of the HTS demultiplexer in cascade with the normal metal power combiner.

It should be noted that the design of the HTS demultiplexer circuit was performed without making any allowances for the use of superconductors since the modeling tools did not exist at the time the design was implemented using Touchstone. Although there did not exist good agreement between the design of each passband's center frequency, the overall passband shape, width, and spacing is reasonably well preserved. Inherent in the logarithmic periodic design is strong interaction between channels due to the manifold design. Partially due to these circuit complexities, the HTS demultiplexer is a good candidate to evaluate the ability of a superconducting microwave circuit design methodology to be useful in the modeling of planar superconducting circuits.

\section{MODELING}

In order to take advantage of the sophisticated microwave circuit CAD software which is commercially available, a method of incorporating the effects of the superconducting film on the parameters of each circuit element is needed. The effect of the superconductor is to introduce an internal inductance, $L_{i}$ (often referred to as the kinetic inductance). Since the conductivity of a superconductor is very large, to first order, the external fields can be presumed to be unchanged from the ideal conducting case for most geometries of practical interest. In comparison to the external inductance, $L_{e}$, in planar microwave circuits, $L_{i}$ is small and the phase velocity, $v_{p}$, and characteristic impedance, $Z_{c}$, are given by:

$$
\begin{aligned}
& v_{p}=\frac{1}{\sqrt{\left(L_{e}+L_{i}\right) C}} \cong v_{p_{e}}\left(1-\frac{L_{i}}{2 L_{e}}\right) \\
& Z_{c}=\sqrt{\frac{L_{e}+L_{i}}{C}} \cong Z_{c_{e}}\left(1+\frac{L_{i}}{2 L_{e}}\right),
\end{aligned}
$$

respectively, where $C$ is the capacitance per unit length. $Z_{c e}$ and $v_{p e}$ are the characteristic impedance and phase velocity, respectively, for the perfectly conducting case. The phase velocity correction can be accommodated by a modified length, $l$, given by

$$
l \cong l_{0}\left(1+\frac{L_{i}}{2 L_{e}}\right)
$$

From a commercially available planar transmission line program such as LineCalc (EESof, Inc.), the microstrip width as a function of characteristic impedance can be curve fit. The fitted function is expanded in a Taylor series which, using (2), is of the form,

$$
w \equiv w_{0}+\frac{Z_{c_{e}} L_{i}}{2 L_{e}} w^{\prime}\left(Z_{c_{e}}\right)+\frac{1}{2}\left(\frac{Z_{c_{e}} L_{i}}{2 L_{e}}\right)^{2} w^{\prime \prime}\left(Z_{c_{e}}\right)+\cdots
$$

around each desired line impedance. The line width as a function of $Z_{c}$ is shown in Fig. 2. Equations (3) and (4) can be incorporated into commercially available microwave CAD software (e.g. Touchstone) once the ratio of $L_{i}$ to $L_{e}$ is defined for each microstrip cross section.

The application of the approximate boundary condition

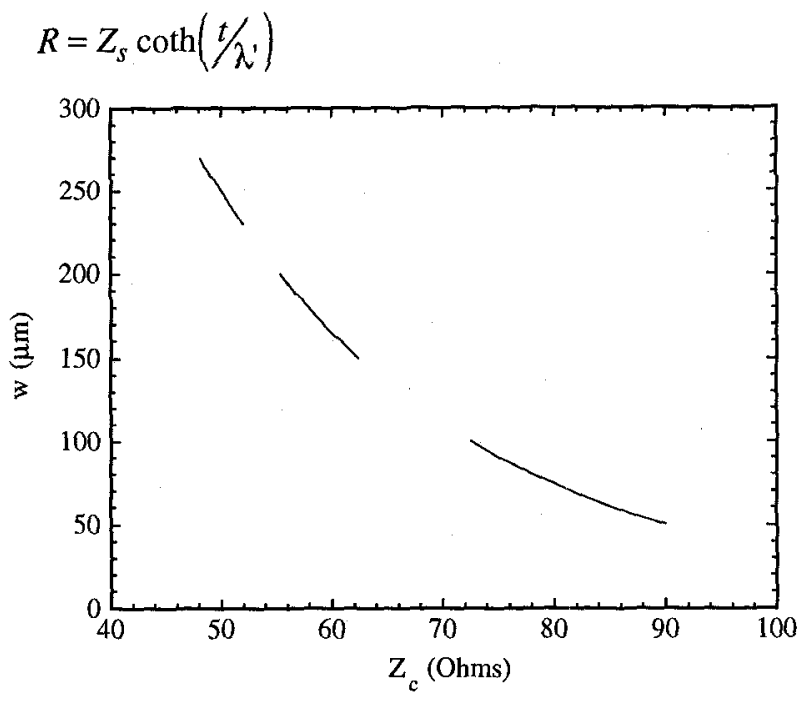

Fig. 2. Dependence of the microstrip width on the characteristic impedance. The dependence was calculated in detail in the vicinity of the three different characteristic impedances used in the demultiplexer design so as to facilitate an accurate Taylor series curve fit for each. 
facilitates the determination of the ratio of the internal to external inductance. In (5), $Z_{S}$ is the surface impedance, $t$ is the superconducting film thickness, and $\lambda^{\prime}$ is the complex penetration depth. The effect of the approximate boundary condition is to replace a finite-thickness region (the superconductor) with a sheet where the internal properties of the region and its thickness are incorporated into the boundary condition [6]. The internal inductance (with a small loss component) is accounted for by the modified boundary condition and the current distribution on the superconductor [6]. The functional form of the current distribution is primarily determined by the cross-sectional geometry of the transmission line and the media. The ratio $L_{i}$ to $L_{e}$ can be accounted for by the product of the approximate boundary condition and a factor which accounts for the cross-sectional geometry of the transmission line. Thus (1) can be written as

$$
v_{p}=\frac{1}{\sqrt{L_{e} C\left(1+\frac{L_{i}}{L_{e}}\right)}}=\frac{1}{\sqrt{L_{e} C\left(1+G t\left(\frac{\lambda}{t}\right) \operatorname{coth}\left(\frac{t}{\lambda}\right)\right)}}
$$

where $G$ is a geometrical factor which takes into account the cross-sectional geometry. Implicit in this formulation is the fact that $G$ is independent of temperature.

Any two-dimensional procedure for determining the propagation constant of planar superconducting transmission lines can be used, assuming the superconducting properties of the film are known. For the circuit studied in detail here, (6) was used to curve fit measured data with a temperature dependence of the penetration depth given by

$$
\lambda(T)=\frac{\lambda_{0}}{\sqrt{1-\left(T / \mathrm{T}_{\mathrm{c}}\right)^{2}}}
$$

which was previously found to be in good agreement with measured data [10], [11]. A best fit was obtained using $T_{\mathrm{c}}=$ $87.6 \mathrm{~K}$ and $\lambda_{0}=135 \mathrm{~nm}$. Employing a spectral domain immittance approach [12], [13], modified to accommodate the approximate boundary condition incorporating the superconducting parameters [6]-[8], yields the desired ratio of $L_{i}$ to $L_{e}$ as a function of microstrip width at the operating frequency of the multiplexer as shown in Fig. 3.

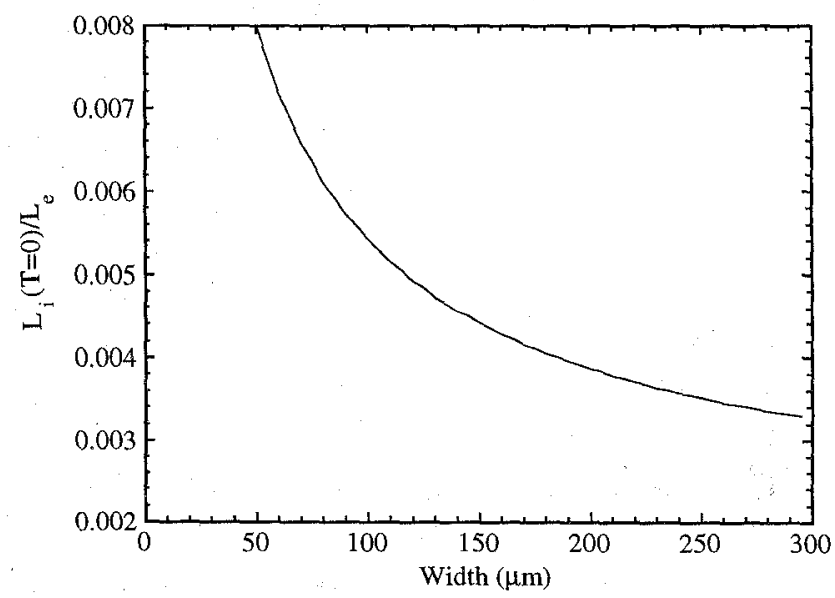

Fig. 3. Calculated ratio of the internal to external inductance at $\mathrm{T}=0 \mathrm{~K}$ for superconducting microstrip transmission lines on a $254 \mu \mathrm{m}$-thick $\mathrm{MgO}$ substrate as a function of the microstrip line width.
Figs. 2 and 3 are parameterized and when joined with (3), (4), and (7) provide equations which can be incorporated into existing commercial microwave circuit $\mathrm{CAD}$ software.

\section{RESULTS}

The methodology described above was incorporated into the Touchstone microwave circuit CAD description of the original demultiplexer design. The power combiner circuitry was also included in the model to be consistent with the measured data. A comparison of the measured results along with the model predictions at $74 \mathrm{~K}, 78 \mathrm{~K}$, and $82 \mathrm{~K}$ are shown in Figs. 4, 5, and 6, respectively. Additionally, the modeled responses of the four HTS demultiplexer channels as de-embedded from the combiner circuit response are also plotted. The right-hand vertical axes are shifted $3 \mathrm{~dB}$ to account for the insertion loss of the combiner circuitry.

The only parameter that was changed between the three simulations shown in Figs. 4, 5, and 6 was the operating temperature. The model does an excellent job of simulating the features of the measured data with very good accuracy. Not only is the relative temperature dependent frequency shift properly accounted for, but the absolute values of the center band frequencies agree remarkably well. Even the out-of-band features are reproduced with good accuracy. It should be noted that the passband ripple increases for the lower frequency channels. This is consistent with the logarithmic periodic design of the demultiplexer. The highest frequency channel is the first channel which is routed from the input manifold. Subsequent channels are tapped off as they progress down the trunk line. Since the original design was done prior to the ability to take into account any effects of the superconductor the element lengths and characteristic impedances are all slightly in error. Those channels which traverse a greater number of these "mistuned" elements will suffer a larger perturbation in their response from the ideal design. These results are sufficient to demonstrate that this approach to modeling complex microwave superconducting circuits has sufficient accuracy to be used as a design tool.

Local variations in the superconducting parameters could account for some of the discrepancies between the measured and the modeled response. Other factors which could contrit

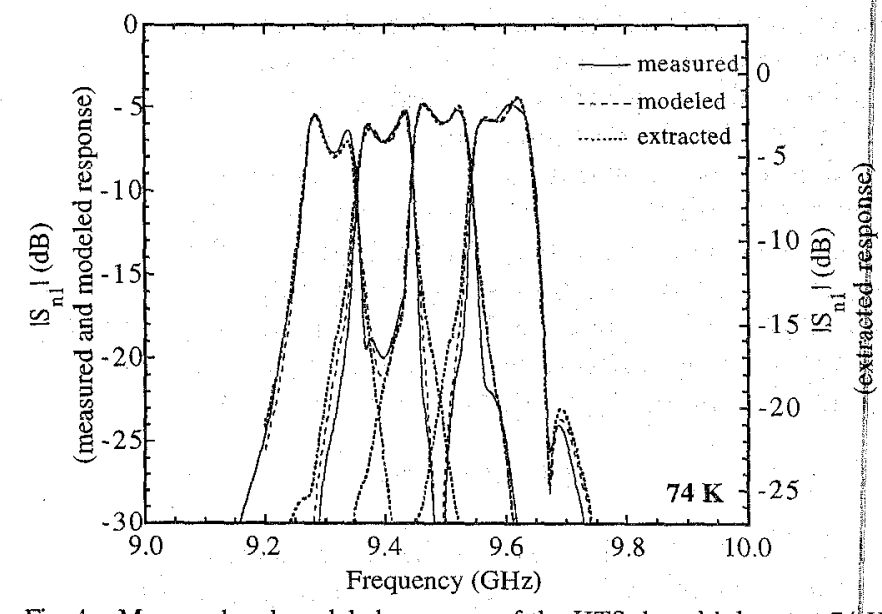

Fig. 4. Measured and modeled response of the HTS demultiplexer at $74 \mathrm{~K}$ in cascade with the normal metal power combiner. Also shown is the deembedded response of the four demultiplexer channels at $74 \mathrm{~K}$. 


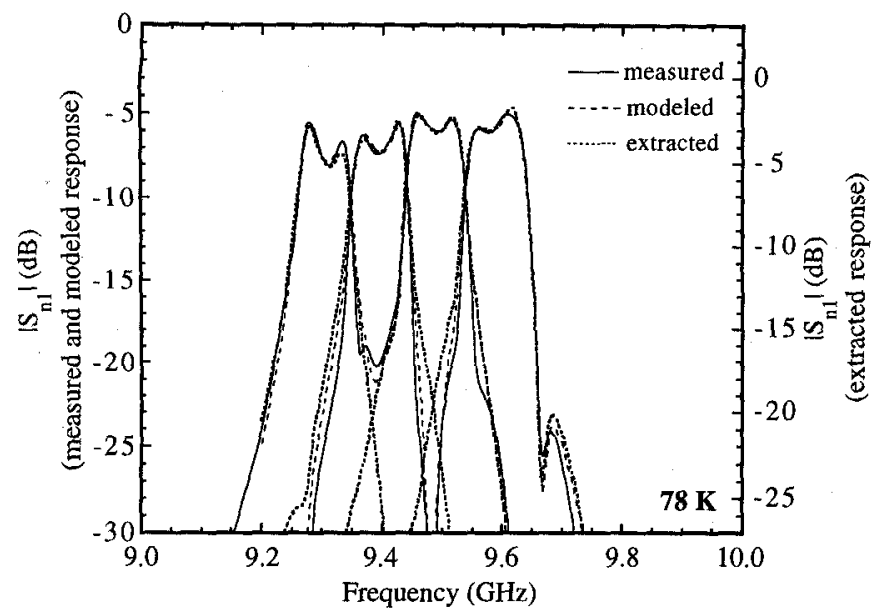

Fig. 5. Measured and modeled response of the HTS demultiplexer at $78 \mathrm{~K}$ in cascade with the normal metal power combiner. Also shown is the deembedded response of the four demultiplexer channels at $78 \mathrm{~K}$.

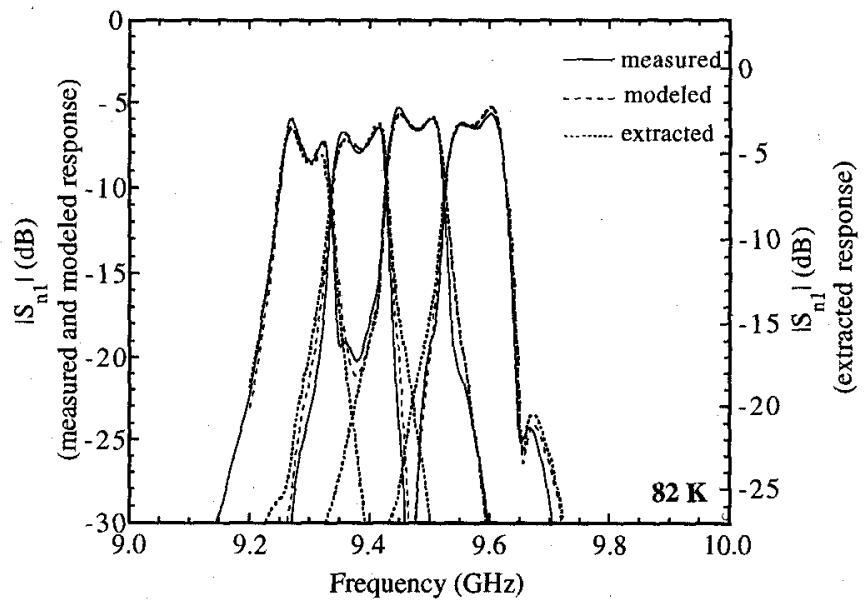

Fig. 6. Measured and modeled response of the HTS demultiplexer at $82 \mathrm{~K}$ in cascade with the normal metal power combiner. Also shown is the deembedded response of the four demultiplexer channels at $82 \mathrm{~K}$.

bute to the differences are patterning and etching variations across different parts of the substrate which will be manifested as perturbations in the $\mathrm{v}_{\mathrm{p}}$ and $Z_{\mathrm{c}}$ from their nominal values.

\section{CONCLUSIONS}

A procedure has been described for accurate and efficient modeling of planar superconducting microwave circuits. The approach utilizes existing microwave circuit CAD software to provide efficient and accurate modeling. A straightforward approach was presented which eases the incorporation of the key affects of superconductivity on the behavior of microwave devices. The primary challenge that superconductivity presents from a microwave design standpoint is the incorporation of the internal inductance into circuit model elements. It was shown that selective use of electromagnetic modeling codes can provide parameterized information quantifying the internal inductance contribution. These corrections were incorporated into conventional microwave circuit CAD software in a straightforward fashion. An excel- lent agreement between measured and modeled data was obtained when this approach was applied to the superconducting demultiplexer portion of the cryogenic channelized receiver delivered by NRL to the HTSSE program.

It should be emphasized that the approach presented here is not dependent on any particular model for the functional dependence of the penetration depth. Nor is it necessary to invoke any particular numerical scheme when determining the internal inductance contributions. Often the geometry of the problem favors the application of a particular approach for efficient calculation of the fields responsible for the internal inductance contribution. This approach provides great flexibility in using modeling tools which are optimized for different situations.

\section{ACKNOWLEDGMENTS}

The authors would like to thank Drs. C. Rauscher and M. Nisenoff for helpful discussions.

\section{REFERENCES}

[1] A Fathy, D. Kalokitis, V. Pendrick, E. Belohoubek, A. Pique, and M. Mathur, "Superconducting narrow band pass filters for advanced multiplexers," 1993 IEEE MTT-S International Microwave Symposium Digest, vol. 3, pp. 1277-1280, June 1993.

[2] L. H. Lee, S. M. Ali, and W. G. Lyons, "Full-wave characterization of high-Tc superconducting transmission lines," IEEE Trans. Appl. Superconduct., vol. 2, pp. 49-57, June 1992.

[3] W. Heinrich, "Mode-matching approach for superconducting planar transmission lines including finite conductor thickness", $I E E E$ Microwave and Guided Wave Letters, vol. 1, no. 10, pp. 294-296, Oct. 1991.

[4] S. El-Ghazaly, R. B. Hammond, and T. Itoh, "Analysis of superconducting microwave structures: application to microstrip lines," IEEE Trans. on Microwave Theory and Tech., vol. 40, no. 3, pp. 499-508, March 1992

[5] W. Schroeder and I Wolff, "Full-wave loss analysis of normal- and superconducting transmission lines by hybrid-mode boundary integral equation method," 1991 IEEE MTT-S International Microwave Symposium Digest, vol. 1, pp. 341-344, June 1991.

[6] J.M. Pond, C.M. Krowne and W.L. Carter, "On the application of complex resistive boundary conditions to model transmission lines consisting of very thin superconductors," IEEE Trans. on Microwave Theory and Tech., vol. 37, no. 1, pp. 181-190, Jan. 1989.

[7] L. H. Lee, S. M. Ali, W. G. Lyons, R. S. Withers, and T. P. Orlando, "Full-wave analysis of superconducting microstrip lines on sapphire substrates," 1993 IEEE MTT-S International Microwave Symposium Digest, vol. 1, pp. 385-388, June 1993.

[8] D. Kinowski, F. Huret, P. Pribetich, and P. Kennis, "Spectral domain analysis of coplanar superconducting line laid on multilayered GaAs substrate," Electronics Letters, vol. 25, no. 12, pp. 788-789, 1989.

[9] C. Rauscher, J.M. Pond, and G.B. Tait, "Cryogenic microwave channelized receiver," IEEE Trans. Microwave Theory and Tech., vol. 44, no. 7, pp. 1240-1247, July 1996

[10] J.M. Pond, K.R. Carroll, J.S. Horwitz, D.B. Chrisey, M.S. Osofsky, and V.C. Cestone, "Penetration depth and microwave loss measurements with a $\mathrm{YBa}_{2} \mathrm{Cu}_{3} \mathrm{O}_{7-\delta} / \mathrm{LaAlO}_{3} / \mathrm{YBa}_{2} \mathrm{Cu}_{3} \mathrm{O}_{7-\delta}$ trilayer transmission line," Appl. Phys. Lett., vol. 59, no. 23, pp. 3033-3035, Dec. 2, 1991 .

[11] A. Z. Kain, J. M. Pond, H. R. Fetterman, and C. M. Jackson, "Impact of kinetic inductance effect on high- $T_{c}$ superconducting coplanar waveguide resonators," Microwave and Optical Technology Letters, vol. 6, no. 13 , pp. 755-758, October 1993

[12] T. Itoh and R. Mittra, "Spectral-domain approach for calculating the dispersion characteristics of microstrip lines," IEEE Trans. Microwave Theory Tech., vol. 21, pp. 496-499, July 1973.

[13] T. Itoh, "Spectral-domain immittance approach for dispersion characteristics of generalized printed transmission lines," IEEE Trans. Microwave Theory Tech., vol. 28, pp. 733-736, July 1980. 